way (that is, to well beyond the transition-state distance); and thus the shift could here have no kinetic influence.

While recognizing that marginal situations arise, we can divide the reactions discussed into four main classes, as illustrated by the schematic energyreaction curves of Figs. 1 and 2. In unaccelerated unimolecular reactions, whether or not they involve a Wagner rearrangement, the 'first ion', and the transition state of its formation, are unaffected by the existence, or possibility of existence, of the 'second ion'. We assume that in unaccelerated Wagner changes there must be two ions; but that when, in unaccelerated reactions, there are two, they are separated by an energy barrier substantial enough to preclude their energetically significant interaction. So we have either (case 1, nN) (the letters mean 'normal rate, normal structure') nonrearranging unimolecular reactions without synartetic acceleration (Fig. 1, broken line), or (case 2, $n W)$ ('normal rate, Wagner change') Wagner rearrangements without such acceleration (Fig. 2, broken line), according as the transition state of the heterolysis which forms the 'first ion' lies energetically lower or higher than that of the co-ordination which destroys or would destroy the 'second ion'.

The other two classes can be most simply described by assuming that there is no resonancestopping barrier between the first and second ionic structures, which interact according to a suggestion ${ }^{10}$ made earlier, to produce a normal ion mor $\ni$ stable than either-a synartetic ion, as we may call it, with a split single bond fastening together the locations of a split ionic charge. We then have not only a stabilized ion, but also stabilized transition states of ionization, and therefore either (case 3,aN) ('accelerated, normal structure') non-rearranging unimolecular reactions with synartetic acceleration (Fig. 1, full line), or (case 4, $a W$ ) ('accelerated, Wagner change') Wagner rearrangements with such acceleration (Fig. 2, full line), according, as before, to which of the two transition states lies lower. For these cases, we should modify our previous formulation:

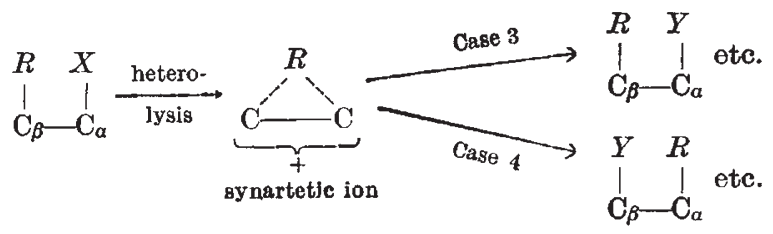

Marginal cases (between 1 and 3 , and between 2 and 4) should arise when a tunnel effect permits weak resonance between the first and second ions ; but we shall not now discuss this phenomenon. Marginal cases of another sort (between 1 and 2 , and between 3 and 4) arise when the two transition states lie on a common energy-level, so that the diagrams of Figs. 1 and 2 become symmetrical, as in Fig. 3. This happens when the unrearranged and Wagner-rearranged products are identical, but for some non-energetic distinction, as when they are enantiomeric, or are labelled isotopically. We then have either (bordercase $12, n N W$ ) reactions without synartetic accelerations (Fig. 3, broken line), or (border case 34, $a N W$ ) reactions with synartetic acceleration, leading, in either case, to 50 per cent of Wagner-rearranged product (for example, to racernization when the optical distinction is employed). A normal-rate

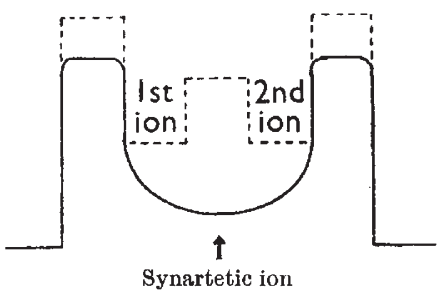

Fig. 3

(The reaction path enters from the left, then divides, and leaves equally by the left and by the right) Border cases 1, 2 (broken line) and 3,4 (full line)

reaction having the described symmetry (border case 12) has been found by Cram ${ }^{11}$ in the solvolysis with racemization (at both asymmetric centres) of that form of 2-phenyl-3- $n$-butyl $p$-toluenesulphonate for which the normal and rearranged substitution products would be enantiomers.

One usually thinks of CC-bond $\sigma$-electrons as having a much smaller tendency to enter into other orbitals than have CH-bond $\sigma$-electrons, or of course $\pi$-bond-electrons or unshared $p$-electrons. Yet it appears that CC-bond $\sigma$-electrons can interact strongly with a carbonium ionic centre to form what we have called a synartetic ion, at least when the stereoelectronic situation is favourable, and when the normal types of conjugation and hyperconjugation are more or less restricted. This seems somewhat less strange when we recall that the carbonium ion has the $\operatorname{sam} \theta$ electron configuration as the tercovalent boron atom, and that it is one of the lessons of boron chemistry that all low-lying orbitals will somehow achieve partial occupation, even when there are too few electrons to occupy them fully (this was recently pointed out to us by Dr. S. H. Bauer) : the same principle may apply in carbonium ion chemistry.

[Feb. 2.

1 Dostrovsky, Hughes and Ingold, J. Chem. Soc., 192 (1946).

Brown, Davies, "Dostrovsky, Evans and Hughes, Nature, [167, 987 (1951)].

${ }^{s}$ Results of present authors.

4 Dostrovsky and Hughes, J. Chem. Soc., 164, 166, I71 (1946).

- Martin, thesis (London, 1949); Nature, 166, 679 (1950).

- Unpublished experiments by Mrs. M. G. Peeling.

7 Nevell, de Salas and Wilson, J. Chem. Soc., 1188 (1939).

- Bateman, Cooper, Hughes and Ingold, J. Chem. Soc., 930 (1940).

- Charlton, Dostrovsky and Hughes, Nature, [167, 986 (1951)]

${ }^{10}$ Ingold, cited by Watson, Ann. Rep. Chem. Soc., 36, 197 (1939), and "Modern Theories of Organic Chemistry", Oxford Press, 2nd Ed,' p. 208 (1941); also by Simonsen and Owen, "The Terpenes", Cram, J. Amer. Chem. Soc., 71, 3836 (1949).

\section{DIFFRACTION GRATINGS}

7 HE Optical Group of the Physical Society held 1 a symposium on diffraction gratings at the Imperial College of Science and Technology, London, on May 10. About seventy-six members and visitors attended and the meeting was generally very successful. A large number of exhibits and demonstrations illustrating the ruling and testing of gratings was shown.

Prof. W. E. Curtis, in introducing the subject, surveyed the present field of application of the grating and indicated the directions in which development is desirable. He mentioned a variety of problems in both atomic and molecular spectroscopy, for which gratings are required, in addition to spectrochemical 
investigations of a more routine nature, which are steadily increasing in number. He pointed out that for these various purposes a wide range of gratings would be required, particularly in regard to spacing and form of the rulings. He remarked, in passing, that it would be more appropriate to specify the spacing directly in microns, rather than as so many lines per inch, as hitherto. He also urged that the present custom of describing performance in terms of 'resolving power', $\lambda / \delta \lambda$, should be abandoned as neither physically significant nor practically convenient, and replaced by a statement of the limit of resolution (based on the Rayleigh criterion) in $\mathrm{cm} .^{-1}$. This has the advantage of depending only on the ruled width of the grating and its setting, and not on the spacing or the spectral region. Its minimum value would be the reciprocal of twice the grating width, as in the reflecting echelon. The optimum spacing in a particular case would be determined by other considerations, such as the overlapping of neighbouring orders and the extent to. which groove shape could be controlled.

Prof. Curtis then referred to some special modifications which may prove valuable in the future, notably the Harrison echelle grating. This has yet to prove itself, but certainly appears very promising. The combination of grating and mirror in Littrow fashion, as proposed by Hulthén and Lind, may well prove valuable in certain circumstances. He remarked also that the introduction of the photomultiplier renders a plane-grating - concave-mirror spectrograph an attractive proposition by reason of its simplicity of construction and adjustment.

So far as ordinary gratings are concerned, Prof. Curtis considers that there are four main directions in which improvement should be sought: the elimination of ghosts, either by perfecting the traditional ruling-method or by developing entirely new ones; the concentration of light in a given direction by control of groove form ; the provision of larger gratings, although a greater ruled width only would suffice for some purposes; and finally, a substantial reduction in cost, so that a spectroscopist can acquire a number of gratings from which he may select the one best suited to each problem. Having regard to the effort already expended on the ruling of gratings, it might seem that only limited progress along these lines would be possible; but modern advances in technique and materials are such as to give good grounds for optimism.

Speaking of the history of the diffraction grating, J. S. Clark said that it originated in the pioneer work of Joseph Fraunhofer early in the nineteenth century. Reference was made to Nobert, of Barth in Germany (1850), and to Rutherford in the United States (1875), who was the first to rule a reflexion grating on speculum metal. The ruling of gratings at Johns Hopkins University, Baltimore, was initiated by Rowland in 1880 and continued by Anderson (1909), Wood (1923) and Strong. Other grating-ruling engines established in the United States were those of Michelson (1900) and Gale (University of Chicago) and those of Anderson (1916) and Babcock (Mount Wilson Observatory).

The Blythswood ruling engine, which has been in the possession of the National Physical Laboratory, Teddington, since 1908, dates from about 1880, whon its construction was undertaken by Lord Blythswood with the assistance of Otto Hilger (one of the founders of the firm of Adam Hilger). Its subsequent history was described by Mr. Clark, and brief mention was also made of the ruling engines constructed by Grayson (1893) at Melbourne and by Siegbahn (1930) at Uppsala and at Stockholm. After pointing out that all existing ruling engines of conventional design have been either of the shaper type (for example, Rowland and Blythswood machines) or of the planer type (such as Strong's new machine at Johns Hopkins University), Mr. Clark went on to discuss the future prospects of ruling large gratings by conventional methods and concluded with a fairly detailed description of the Blythswood machine in its present state.

J. Guild described how interference tests, which have long been familiar in their application to the examination of mirrors, prisms, lenses and even complete optical instruments, can be applied to diffraction gratings, and stressed the fundamental importance of these tests in assessing the optical performance of gratings and indicating the nature, magnitude and location on the grating surface of the defects of figure or errors of ruling responsible for defects of performance.

The tests described were an adaptation of the well-known Fizeau test for plane mirrors to plane reflecting gratings : an adaptation of the differential interference (sheared wave-front) test developed by Ronchi and Bates for concave mirrors to either concave or plane gratings ; and a test depending on the interference between the wave-fronts diffracted by a superposed pair of transparent replicas of a plane grating. For the second test Mr. Guild employed a simple beam-dividing device based on the Wollaston or Rochon double-image prism. This is free from some of the defects of Ronchi's apparatus and, while possessing all the flexibility required for the present purpose, is much simpler and less costly than the wave-front shearing interferometer of Bates. Slides were shown of interferograms obtained by each of these methods, those illustrating the first two being of gratings ruled on the Blythswood, Rowland and Siegbahn engines.

It was clear from those examples that, judged by ordinary optical standards, there is plenty of room for improvement in the best gratings produced by ruling engines of the traditional types. It was also apparent that the makers of gxatings should be more careful than they seem to have been to rule only on blanks of good optical quality.

Dr. L. A. Sayce began by pointing out that diffraction gratings made by the traditional methods laid down by Rowland have certain defects, some inherent in all reciprocating engines and some attributable to the residual errors of screws, division plates, thrust bearings and the like. There is therefore special interest in a new approach to the problem suggested by Sir Thomas Merton which is being developed in the National Physical Laboratory by the Light and Metrology Divisions. Dr. Sayce explained that the fundamental concept is to rule diffraction gratings upon cylinders. Such helical gratings can be used directly in conjunction with cylindrical lenses or mirrors, but can more conveniently be used as matrices for the preparation of plane replicas of which many identical 'copies' can be made from each original ruling.

Using this principle, therefore, the production of a plane grating now consists of three main stages. In the first, a screw of up to 15,000 threads per inch is cut upon one-half of a metal cylinder using a suitably modified lathe. In the second, this lathe-cut thread is copied upon the other half of the cylinder 
using a 'Merton nut' to remove its inevitable periodic errors. In the third stage, the corrected helix is covered with a thin layer of plastic which, when hardened and annealed, is removed from the cylinder and opened out upon an optically flat surface of swollen gelatine. When the whole has dried, the plastic 'pellicle' can be removed, and the underlying gelatine is found to be moulded into a flat replica of the corrected helix and can be hardened and aluminized.

It was shown by means of specimens and interferograms that these methods have already produced satisfactory gratings, particularly for work in the infra-red, and there seems every reason to hope that they will in due course yield large ghost-free gratings suitable also for emission spectroscopy. As it has been found impossible to devise a method of producing concave gratings from cylindrical matrices, a machine has been developed at the National Physical Laboratory for applying to a reciprocating concave blank a tool advanced by a 'Merton nut' driven by a screw of the pitch of the required grating.

Dr. A. C. Menzies, after referring to some recent work by Messrs. Hilger-Watts on the construction of fine-mesh metallic grids, went on to describe a modification of Sir Thomas Merton's process with the object of producing long gratings. This employs three successive operations. In the first, a short length of fine-pitch thread is engraved on a metal cylinder $B$ by a diamond driven from a 'Merton nut' riding on a similar cylinder $A$ on which a coarse thread has been engraved by a lathe. The two cylinders are connected together by a gear of ratio $32: 1$. In the second stage, a 'Merton nut' on $B$, with a diamond just ahead of it, extends the fine pitch thread as far as desired along the cylinder. The passage of the nut over the thread which it has ruled tends to produce irregular burnishing of the rulings. Although this would probably have little effect on the performance of a replica taken from the cylinder, it may be eliminated by ruling a third cylinder $C$ by means of a 'Merton nut' riding on $B$, the two cylinders being coupled together by a $1: 1$ gear. This cannot introduce periodic error, although it may give rise to slight non-linearity of the rulings, which, however, is regularly repeated and therefore not objectionable. In all cases, one end-bearing consists of a "Merton nut' on a 'neutral' thread, and the other of a similar nut on polished bar. A steel bar ruled by this method for a length of about nine inches was exhibited.

Dr. Menzies also directed attention to the probable value of the 'superfinishing' process, as used by Chryslers in the United States, in much reducing the time required to polish the metal cylinders preparatory to ruling by the Merton method.

In the discussion which followed, F. Twyman emphasized the desirability of comparative testing of existing gratings, especially some of the older ones reputed to be of 'good' quality. Prof. Curtis asked whether any correlation has yet been attempted between the results of the tests described by Mr. Guild and the performance of the grating in an actual spectrograph, to which Mr. Guild replied that this has not yet been possible as sufficient gratings of various qualities have not yet been available. Mr. Guild said that it is his object to establish such a correlation, and added that, although the tests might be capable of disclosing defects which are too slight to affect spectroscopic performance, such additional sensitivity is of great advantage in monitoring the development of the ruling process.
G. Sissen asked Mr. Guild whether it is possible by his wave-front shearing method to alter the inclination of the interfering wave-fronts, and consequently the spacing of the fringes. The latter replied that variation of fringe spacing is effected by displacing the double-image prism along the axis of the instrument. The direction of the fringes is, however, fixed relative to the direction of shear, in which respect Mr. Guild's beam divider is less flexible than that of Bates for general optical testing. Mr. Sissen then asked Dr. Sayce whether the thickness of the gelatine layer in the third stage of his process could be sufficiently increased to produce infra-red gratings, and received the reply that, although an increase in the thickness of the layer causes a slight departure from flatness, this is not enough to be detrimental in the infra-red region.

There was some discussion of the possibilities of the step-by-step method of grating ruling, which is attractive by reason of the elimination of the screw and its attendant errors. Various speakers referred to attempts on these lines, made in Germany, Sweden and Great Britain ; but it appears that none has been successful. The fundamental difficulty is thought to be that in any process of this type an accidental error of spacing will necessarily affect the whole of the subsequent rulings.

\section{THE 1951 EXHIBITION OF SCIENCE}

$T$

HE 1951 Exhibition of Science is splendidly housed, to the south of the Imperial College, in an extension of the Science Museum which is to form the nucleus of new permanent additions to the Museum buildings. A wealth of skill and decorative genius has gone to the making of a display which, despite some minor shortcomings, is an outstandingly good presentation of some of the main developments of pure science during recent years, with some appropriate historical background. It is planned as a continuous narrative (one might almost say a short course) and the round trip takes between one and two hours.

One enters by a maze-like tunnel with soundproofed bays, in each of which a loud-speaker suggests that one is shrinking to atomic dimensions as illuminated models of graphite crystals expand successively; at the end of the tunnel the visitor is on equal terms with an electron cloud. He is to see modern science from the atomic point of view.

The first stage of the journey around the exhibition itself deals with atomic structure. The diagrams and mural showing the history of the Periodic Table and its modern representation are good. The experiments on the electron are indifferent, chiefly due to lack of explanation as to why electric and magnetic deflexions occur ; and some of those listed are missing. There are some excellent animated diagrams illustrating radioactivity and nuclear physics, and two cloud chambers which work beautifully; but why the catalogue should include under radioactivity a spectrometer "capable of refractive index measurements of $0 \cdot 000001$ ", whatever that may mean, is a mystery.

Next come sections on chemical structure, the nature of chemical bonds, and the structure of metals and crystals. These are on the whole mag- 